\title{
MIDDLE- AND FAR-ULTRAVIOLET OBSERVATIONS OF HOT WHITE DWARFS
}

\author{
S. B. Parsons, J. D. Wray, and K. G. Henize \\ Department of Astronomy \\ University of Texas at Austin
}

\section{INTRODUCTION}

Several hot subluminous stars were first recognized as such from low-resolution spectra obtained with the ultraviolet objective-prism survey from Skylab, Experiment S-019. About 9 percent of the sky was photographed, 3 percent with unwidened spectra. Two hot subdwarf stars have been reported previously, HDE 283048 (Laget et al. 1978) and the companion to HR 3080 (Parsons et al. 1976a). Papers on two other subdwarfs are in preparation. One previously unrecognized white dwarf star was found, HD 149499 B, and new observations (Wray, Parsons and Henize 1979) show it to be probably the hottest white dwarf known.

\section{HD 149499 B}

HD 149499 is composed of a KO V primary (Houk and Cowley 1975) with a secondary 3 mag fainter separated by about 2" (Rossiter 1955). The companion was discovered to be most probably a hot white dwarf in one of the fields obtained in 1973 with the Experiment S-019 ultraviolet spectrograph (Parsons et al. 1976b). Wegner (1979) has reported ground-based spectral and photometric observations of HD $149499 \mathrm{AB}$ which confirm the nature of the secondary. A nearly uncontaminated spectrum of 149499 B shows a possible broad He II $\lambda 4686$ line but no hydrogen Balmer lines. The photometric data indicate a temperature in excess of $50,000 \mathrm{~K}$ and perhaps hotter than $10^{5} \mathrm{~K}$. He suggests that HD $149499 \mathrm{~B}$ is similar to the helium-rich DO star HZ 21.

One of us (JDW) has obtained observations with the short wavelength (SWP) and long wavelength (LWR) cameras of the International Ultraviolet Explorer (IUE) on 5 February 1979. Two exposures were made with each camera; all exposures were unwidened and were obtained with the large 10" $\times 20^{\prime \prime}$ aperture to ensure recording all of the light from both components. The long exposures ( $5 \mathrm{~min}$, SWP 4165, and $8 \mathrm{~min}$, LWR 3686) were partially overexposed. The short exposures (100 sec, SWP 4166, and $4 \mathrm{~min}$, LWR 3687 ) were optimum. 
Routines have been developed at the University of Texas by one of us (SBP) to convert the IUE intensities to fluxes and plot them at any desired scale. We have adopted the preliminary flux calibration of IUE (Bohlin and Snijders 1978) which is apparently accurate to 10 or $15 \%$. The four spectra of $149499 \mathrm{AB}$ have been averaged together and show a very steep energy distribution. The KO dwarf probably contributes a significant amount of flux toward the long wavelength end but should not affect the flux shortward of about $2200 \AA$.

Absorption lines of moderate strength are seen at He II $\lambda 1640$ and at $\lambda 1216$. Several lines of the He II $\lambda 46863 \rightarrow n$ series are visible in the middle UV. The $\lambda 1216$ line is of particular interest in view of Wegner's suggestion that this star may be helium rich (i.e. hydrogen poor). Although He II $\lambda 1215$ is undoubtedly a significant contributor to this feature, the observed strength is slightly greater than that of $\lambda 1640$ and strongly suggests that weak hydrogen Ly $\alpha$ is also present. Over the estimated distance of $30 \mathrm{pc}$ to the system, interstellar Ly $\alpha$ is unlikely. Comparison of the HD 149499 B ultraviolet spectrum with that of $\mathrm{HZ} 21$ published by Greenstein and Oke (1979) indeed indicates that more hydrogen is present in HD 149499 B than in $\mathrm{HZ} \mathrm{21,} \mathrm{the} \mathrm{prototype} \mathrm{DO} \mathrm{star.} \mathrm{On} \mathrm{the} \mathrm{other} \mathrm{hand} \mathrm{Wegner's}$ inability to observe the Balmer lines indicates that this hydrogen abundance is significantly less than in the DA stars. Since the value of $\log \mathrm{N}_{\mathrm{He}} / \mathrm{N}_{\mathrm{H}}$ in $\mathrm{HZ} 21$ derived by Koester, Liebert, and Hege (1979) is roughly 0.8 , it appears that $\mathrm{He} / \mathrm{H} \geq 1$ for $\mathrm{HD} 149499 \mathrm{~B}$.

We note that analysis of the strengths of He II lines arising from two different levels should provide an excitation temperature for HD $149499 \mathrm{~B}$. This analysis as well as a firmer estimate of hydrogen abundance is in progress.

The number of degenerate stars known with temperatures above $50,000 \mathrm{~K}$ is extremely small. Greenstein and Oke (1979) present IUE data on the UV fluxes of two of the hotter white dwarfs, HZ 43 (DAwk) and $\mathrm{HZ} 21$ (DO). Comparison with model atmospheres with appropriate $\log \mathrm{g}$ indicates temperatures of 60,000 and $50,000 \mathrm{~K}$ respectively. Wesselius and Koester (1978) compared ANS satellite ultraviolet photometry of ten hot DA stars with model atmospheres and find effective temperatures of about $62,000 \mathrm{~K}$ for the two hottest in the sample, $\mathrm{HZ}$ 43 and Feige 24.

To aid in deriving a temperature for HD 149499 B, Koester (1979) has kindly supplied fluxes for atmospheres as described by Koester et al. (1979) with $\log g=8, \mathrm{He} / \mathrm{H}=0.1,10$, and 100 , and $\mathrm{T}_{\text {eff }}=60$, 75 , and $100,000 \mathrm{~K}$. Although a model with $\mathrm{He} / \mathrm{H}=10, \mathrm{~T}_{\text {eff }}=100,000 \mathrm{~K}$ was not available, the other models having $\mathrm{He} / \mathrm{H}=10$ and 100 have virtually identical fluxes at the same temperature values. The left side of figure 1 shows the fit of $\mathrm{He} / \mathrm{H}=100$ models to the HD $149499 \mathrm{~B}$ data. The quantity plotted is the logarithm of the ratio of the stellar flux (F) to the model flux ( $f=$ surface $f l u x / \pi$ ) at each wavelength. A constant ratio would imply a good temperature fit. The white dwarf component is clearly hotter than $60,000 \mathrm{~K}$ and probably hotter than $75,000 \mathrm{~K}$, but appears slightly cooler than $100,000 \mathrm{~K}$. A 
graphical interpolation of the slopes yields an estimated temperature of $93,000 \mathrm{~K}$ for HD $149499 \mathrm{~B}$.

These values are sensitive to any wavelength dependent error which may be found in the IUE flux calibration (cf. Greenstein and Oke 1979). It is of interest to compare also the visual magnitude data of Rossiter and Wegner with the model atmosphere data. $V=11.71 \pm 0.2$ is obtained for the white dwarf if we combine Rossiter's $\Delta \mathrm{m}=2.95$ (the mean of two measures, 2.8 and 3.1) with Wegner's combined $V$ magnitude of 8.69. The error is the estimated maximum error in Rossiter's visual $\Delta \mathrm{m}$. This magnitude for component $B$, after conversion to a flux value and division by the model atmosphere flux at $5500 \AA$, is displayed on the right side of figure 1 for each of the three temperatures. The lines join the visual data with the IUE data at $1500 \AA$. Again, a horizontal line, $1 . e$. a constant flux ratio, indicates the best fit. In this case the best fit occurs at about $82,000 \mathrm{~K}$.

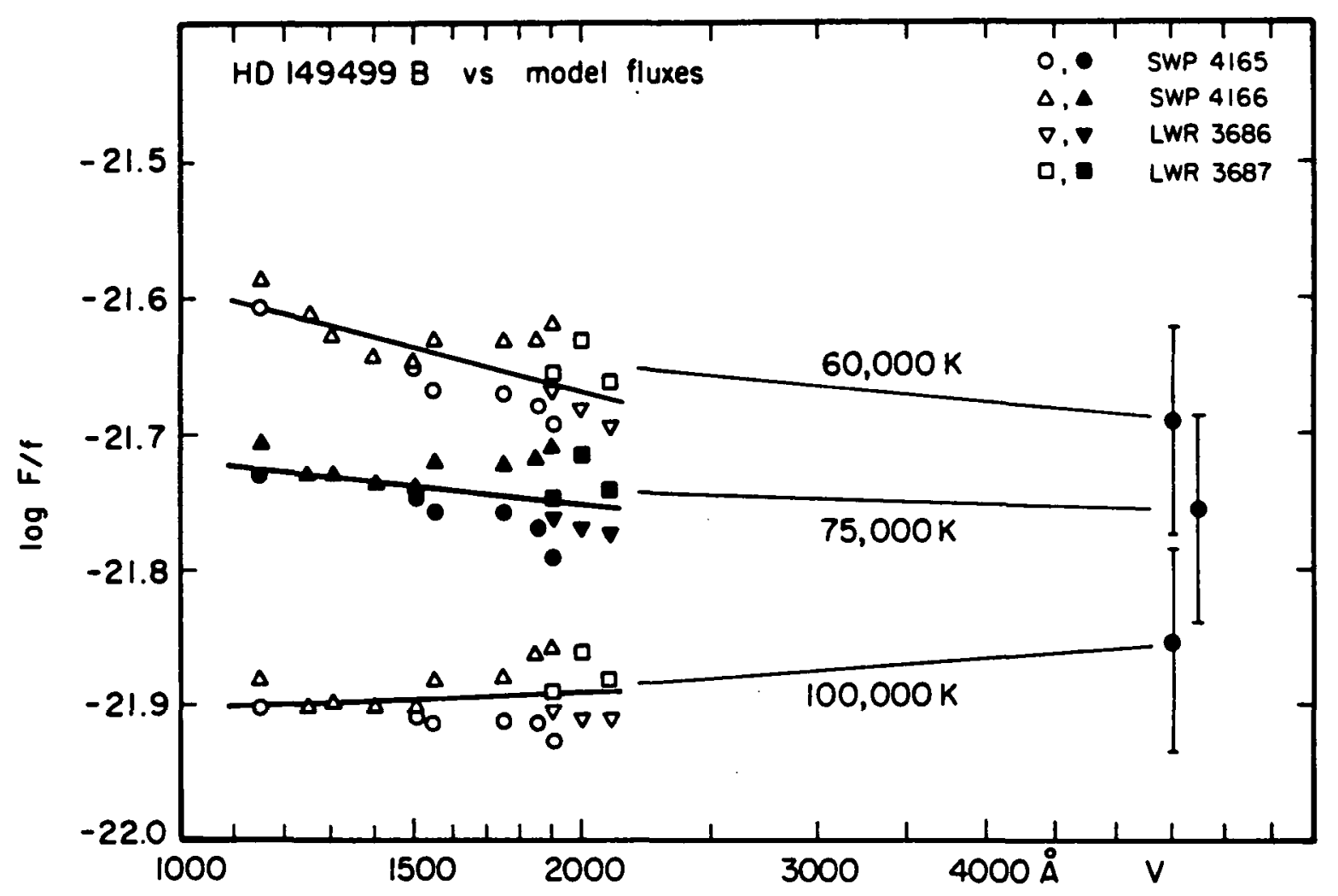

Fig. 1 - Comparison of observed HD 149499 B fluxes with computed fluxes from He-rich model atmospheres (Koester 1979). Symbols on left represent continuum fluxes at selected wavelengths from the designated IUE exposures, divided by model fluxes. At right the visual flux from component $B$, divided by model fluxes, is plotted with error bars representing an uncertainty of \pm 0.2 mag. Results are shown for three of the temperatures for which model atmosphere fluxes are avallable. A logarithmic wavelength scale is used for convenience. 
Which of these estimates is the more accurate? Although the $\lambda 1500$ vs. $V$ comparison is probably subject to greater errors in the two data points, including possible error in the absolute calibration of IUE, the longer wavelength baseline reduces their effect. A reasonable error estimate of these temperatures is $\pm 10,000 \mathrm{~K}$ in both cases.

The above temperature estimates are based on a $\mathrm{He} / \mathrm{H}$ ratio of 10 to 100. A reduction in derived temperature of $10-15,000 \mathrm{~K}$ results if fluxes are used for $\mathrm{He} / \mathrm{H}=0.1$. Since it appears that the He/H ratio of $\mathrm{HD} 149499 \mathrm{~B}$ is nearer to 10 than to 0.1 , we conclude that the remperature reduction due to this factor is somewhat less than $5,000 \mathrm{~K}$.

Thus the IUE data indicate that the temperature of HD 149499 B probably lies between 80,000 and $90,000 \mathrm{~K}$ and almost certainly between 70,000 and $100,000 \mathrm{~K}$. This makes the star the hottest white dwarf star for which reasonably reliable temperature estimates are avaflable. We urge southern hemisphere observers to measure position angles and separations of this binary so that a mass estimate can be made.

\section{FUTURE WORK}

We are hoping to refly Experiment S-019 on Spacelab. With an ultraviolet to optical image converter, two-stage image intensifier, and roll film magazine, the entire sky can be surveyed in two or three missions with unwidened spectra to $m_{v}=14$ (unreddened BO star detected at $2400 \AA$ ). The spatial resolution is about 20 arc sec. We expect to find a dozen or more new hot white dwarfs and hundreds or thousands of hot subdwarfs, giving complete samples over distances of about 100 and $1000 \mathrm{pc}$, respectively.

To allow rapid identification of images, we will be compiling a data base of all known faint blue objects. We will need to compute in advance the expected appearance of the fields. We would appreciate copies of lists of data on white dwarfs and other objects, with coordinates whenever feasible, in order to have as comprehensive a data base as possible. In return, we hope to be able to make the data base available in a few years for general use.

We gratefully acknowledge the assistance of the IUE Observatory staff in the acquisition and reduction of these data. We are grateful to $\mathrm{Dr}$. Koester for providing model-atmosphere flux data. This work 1s supported in part by the National Aeronautics and Space Administration under grants NSG 5337, NSG 7371, and NSG 5328.

\section{REFERENCES}

Bohlin, R.C., and Snijders, M.A.J. 1978, IUE NASA Newsletter, No. 2. Greenstein, J.L., and Oke, J.B. 1979, Astrophys. J. (Letters), 229, 2141.

Houk, N., and Cowley, A.P. 1975, University of Mchigan Catalogue of Two-Dimensional Spectral Types for the HD Stars (Ann Arbor: Lithocrafters, Inc.), Vol. 1. 
Koester, D. 1979, private communication.

Koester, D., Liebert, J., and Hege, E.K. 1979, Astr. Astrophys., 71, 163.

Laget, M., Vuillemin, A., Parsons, S.B., Henize, K.G., and Wray, J.D. 1978, Astrophys. J., 219, 165.

Parsons, S.B., Henize, K.G., Wray, J.D., Benedict, G.F., and Laget, M. 1976b, Astrophys. J. (Letters), 206, L71.

Parsons, S.B., Wray, J.D., Kondo, Y., Henize, K.G., and Benedict, G.F. 1976a, Astrophys. J., 203, 435.

Rossiter, R.A. 1955, Pub. Obs. Univ. Michigan, 11, 1.

Wegner, G. 1979, Mon. Not. Roy. Astr. Soc., 187, 17.

Wesselius, P.R., and Koester, D. 1978, Astr. Astrophys., 70, 745.

Wray, J.D., Parsons, S.B., and Henize, K.G. 1979, submitted to Astrophys. J. (Letters). 